
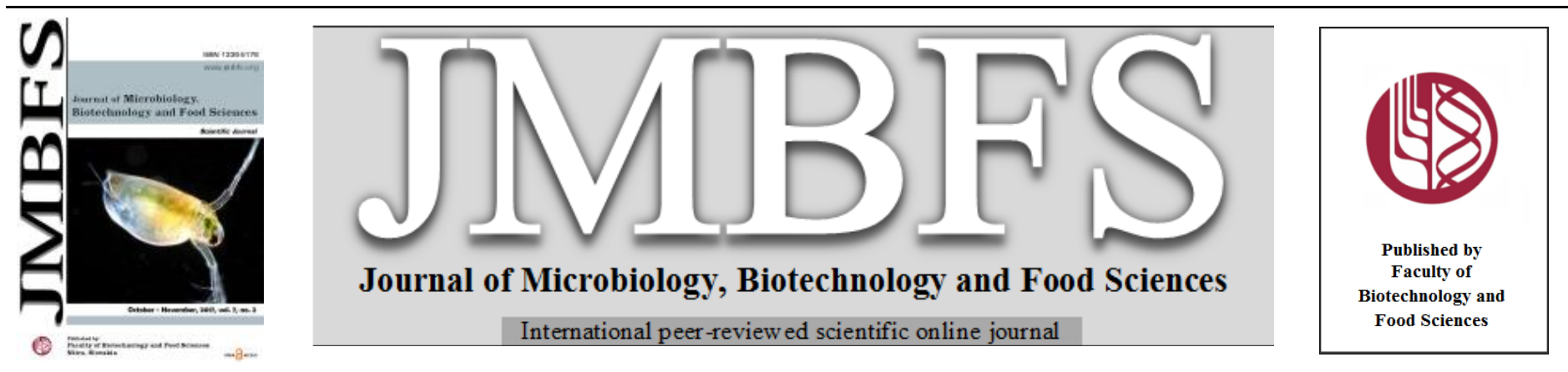

\title{
THE ANTI-MICROBIAL EFFECT OF FOOD WRAP CONTAINING BEESWAX PRODUCTS
}

\section{Crystal T. Pinto ${ }^{1}$, Jarosław A. Pankowski* ${ }^{2}$, Francis E. Nano ${ }^{1}$}

\author{
Address(es): \\ ${ }^{1}$ University of Victoria, Department of Biochemistry and Microbiology, 3800 Finnerty Road, Victoria BC V6P 4W8, Canada. \\ ${ }^{2}$ University of Nebraska-Lincoln, Department of Chemistry, 1400 R Street, Lincoln, NE 68588, USA.
}

*Corresponding author: jaroslaw.pankowski@unl.edu

doi: 10.15414/jmbfs.2017.7.2.145-148

\section{ARTICLE INFO}

Received 14.11.2016

Revised 5. 8. 2017

Accepted 12.9. 2017

Published 1. 10. 2017

Regular article

open ${ }_{\text {ACCESS }}$

\begin{abstract}
Food preservation is a vital issue on a global scale. It reflects both the need to prevent food spoilage as well as the necessity for reduction in the occurrence of food-borne pathogens. Progress of technology allowed for the development of various techniques that inhibit the growth or activity of food contaminating microbes. In the last few years we saw creation of various types of antimicrobial food packaging systems. They contain active antimicrobial agents immobilized on a material that acts as a food wrap. In this paper we have tested the efficiency of Abeego, a commercially available food wrap, against representatives of different groups of microbes. The Abeego wrap contains natural products such as waxes and oils, which can display antimicrobial activity. We analyzed the ability of the wrap and its components to affect the growth and replication of various groups of microorganisms. We have discovered that the Abeego wrap was capable of significantly inhibiting the viable cell count of bacterial species - both gram positive and gram negative. However no noticeable antifungal or antiviral activity has been observed. This implies that Abeego wrap can act as an efficient mean of inhibiting the spread of food-borne bacterial pathogens, as well as contribute to preventing food spoilage.
\end{abstract}

Keywords: food preservation; antimicrobial; food wrap; Salmonella

\section{INTRODUCTION}

The spoilage and contamination of groceries caused by microbes is a serious concern for many branches in the food industry. It results in food products being unsuitable for consumption and can account for significant financial losses. It is estimated that approximately $25 \%$ of world's food supply is lost due to the activity of microorganisms (Singhet al. 2016). Therefore preventing product spoilage in food industry is one of the most vital matters. Diseases caused by food-borne pathogens are another major issue for food distribution. The problem of food preservation has been known since the beginning of civilization and as a result multiple techniques of conserving food have been developed. This includes methods such as drying, salting and pickling. In many cases, especially in modern food preservation, use of antimicrobial agents plays a significant role. Especially the chemical compounds that stop the growth and activity of microbes allow for food to remain consumable for much longer. New possibilities in the field of food preservation have become available thanks to the use of antimicrobial food packaging (Malhotra et al. 2015). This type of packaging material incorporates elements that inhibit the growth of pathogenic bacteria and fungi. In some cases the active agent can be a natural product.

Abeego is a consumer food wrap material that consists of a coated fabric. The main constituent of Abeego is beeswax which has been deemed "generally regarded as safe" (GRAS) ("Select Committee on GRAS Substances (SCOGS) Opinion: Beeswax (yellow or white)," 1975) by the U.S. Food and Drug Administration. Lesser components include tree resin and oils. One of the potential advantages of using beeswax as part of a food wrap material is its antimicrobial properties (Antúnez et al., 2008; de Andrade Ferreira et al., 2007; Orsi et al., 2005; Wilsonet al. 2015; Zhang et al. 2013). The anti-microbial effects of beeswax are attributed to propolis (Marcucci et al., 2001), a sticky material used by bees to seal holes and cracks in beehives. The major constituents of propolis are resins derived from the plants that the bees visit while collecting pollen. The variation in flora in different geographical locations will affect the constituents of propolis (Wilson et al. 2013; 2015) and, in turn, this will affect the potency of the anti-microbial effect of each lot of beeswax. Thus, it is important for manufacturers of food service products using beeswax to have evidence that each lot of beeswax has a minimal level of anti-microbial activity. Presumably, much of the anti-bacterial activity of Abeego wrap comes from the propolis fraction of the beeswax. However there could be contributions from the resin, oil and fabric components as well.

The aim of this project was to investigate the anti-microbial activity of the Abeego wrap and its components. The anti-bacterial activity of Abeego is predominently attributed to the propolis fraction of the beeswax, however there is a possibility of resin, oil and fabric components to play an important role too Therefore we decided to investigate the effect of the Abeego product and its individual components on different groups of microbes. Since food-borne pathogens can be found among bacteria, fungi and viruses, we have investigated representatives of each group. The individual components of Abeego wrap analysed during this project have been taken from the lot used to make the final product.

\section{MATERIAL AND METHODS}

Preparation of the Abeego wrap and its components

The Whatman ${ }^{\mathrm{TM}}$ Filter Paper Circles $(12.7 \mathrm{~mm})$ were used as carriers for the oil and wax. Fragments of Abeego wrap and fabric were cut out to have the same shape and size as paper circles. Papers and wrap were sterilized in the autoclave. Oil was prepared by leaving it in boiling water for 1 hour. The resins were sterilized with $70 \%$ ethanol. The sterile paper circles were dipped in oil or molten wax. For standardization purposes pieces of resins were selected so that their weight would be within $10 \%$ of each other.

\section{Strains and growth conditions}

Bacterial strains of Bacillus cereus, Escherichia coli W3110, Pseudomonas aeruginosa, Salmonella enteritidis and Staphylococcus aureus were used for the anti-bacterial activity experiments. For positive control discs soaked in 
kanamycin $(25 \mu \mathrm{g} / \mathrm{ml}$ or $50 \mu \mathrm{g} / \mathrm{ml})$ were used. Two strains of Saccharomyces cerevisiae - CRY1 and MYA3666 (VL6-48) - were used as representatives of yeasts. Bacteriophages M13 (Messing, 1993) and P1vir (Luria et al. 1960) were used for the viral assays. The M13 phage was amplified by infecting E. coli JM109 strain, while the P1vir was amplified by infecting E. coli DH10B Bacterial broth growth was done in Luria-Bertani (LB) medium (Bertani, 1951) Yeast broth growth was done in the yeast extract-peptone-dextrose (YPED) medium supplemented with adenine (YPAD) (Murthy et al. 1975). For solid media agar was added to concentration of $1.5 \%$.

\section{Zone of inhibition assay}

The strains were grown overnight in LB and undiluted samples were spread on LB plates using sterile cotton swabs. Discs of Abeego wrap, fabric, oil, resin and beeswax were prepared and sterilely placed on the plates with the above cultures. The Abeego fabric was used as a negative control (as it contains no beeswax) and discs soaked in kanamycin were used as positive control. The plates were incubated at $37^{\circ} \mathrm{C}$ for $18-20$ hours until the bacterial lawn of growth was observed. The zones of inhibition were analyzed by measuring their radius i.e. the distance from the center of zone to its border.

\section{Bacteria survival assay}

For $S$. enteritidis assay the Abeego discs were left in $1 \mathrm{ml}$ LB medium in a microtiter plate for 18 hours. Then $100 \mu$ of $S$. enteritidis inoculum $\left(\sim 10^{5} \mathrm{cfu} / \mathrm{ml}\right)$ was added into the wells. The cultures were incubated with shaking at $30^{\circ} \mathrm{C}$ for 24 hours. After that time the cells in each well were enumerated on dilution plates. This experiment was performed in quadruplicates. For $S$. aureus assay the Abeego discs were left in $1 \mathrm{ml} \mathrm{LB}$ medium in a microtiter plate for 72 hours Then $100 \mu \mathrm{l}$ of $S$. aureus inoculum $\left(\sim 10^{6} \mathrm{cfu} / \mathrm{ml}\right)$ was added into the wells. The cultures were incubated with shaking at the room temperature for 40 hours. After that time the cells in each well were enumerated on dilution plates. This experiment was performed in pentaplicates. For both experiments the data was analyzed using unpaired T-test.

\section{Yeast survival assay}

For the yeast experiments the Abeego discs were left in YPAD medium in a microtiter plate for 24 hours. Then inoculum from an overnight $S$. cerevisiae culture $\left(\sim 10^{-6} \mathrm{cfu} / \mathrm{ml}\right)$ was used to start the fresh cultures. The cultures with and without the Abeego wrap were grown at a room temperature for 48 hours. After that time the cells in each well were enumerated on dilution plates. These analyses were performed in triplicates. For this experiment the data was analyzed using unpaired T-test.

\section{Phage activity assay}

For the M13 experiment $\sim 10^{3}$ phage particles were suspended in LB medium in a microtiter plate. The phages were incubated with and without sterile Abeego wrap, at a room temperature with shaking. After 50 hours, plaque assay was carried out by inoculating the phage with the JM109 strain into LB soft agar and creating an overlay. For the P1/vir experiment $\sim 10^{6}$ phage particles were suspended in LB medium in a microtiter plate. The phages were incubated with and without sterile Abeego wrap, at a room temperature with shaking. After 24 hours, plaque assay was carried out by inoculating the phage with the DH10B strain into LB soft agar and creating an overlay.

\section{RESULTS AND DISCUSSION}

\section{Growth inhibition assay on agar plates}

To test the spectrum of anti-bacterial activity of the commercial products, a zone of inhibition assay was carried out against the bacteria that are some of the most common food-borne pathogens and environmental contaminants. The zone of inhibition assay was utilized as it allows for a quick determination of an inhibitory effect of a given substance. The impact of Abeego wrap and its components was compared to a positive control in form of antibiotic discs. After the incubation no zone of inhibition was observed with Abeego fabric, beeswax, oil and resin against any of the analysed bacteria. At the same time the positive control generated visible zone of inhibition (Fig 1). The likely explanation for this lack of effect from the tested products is the limited diffusion into the surrounding medium. Therefore in order to properly asses the activity we had to utilize an alternative method.

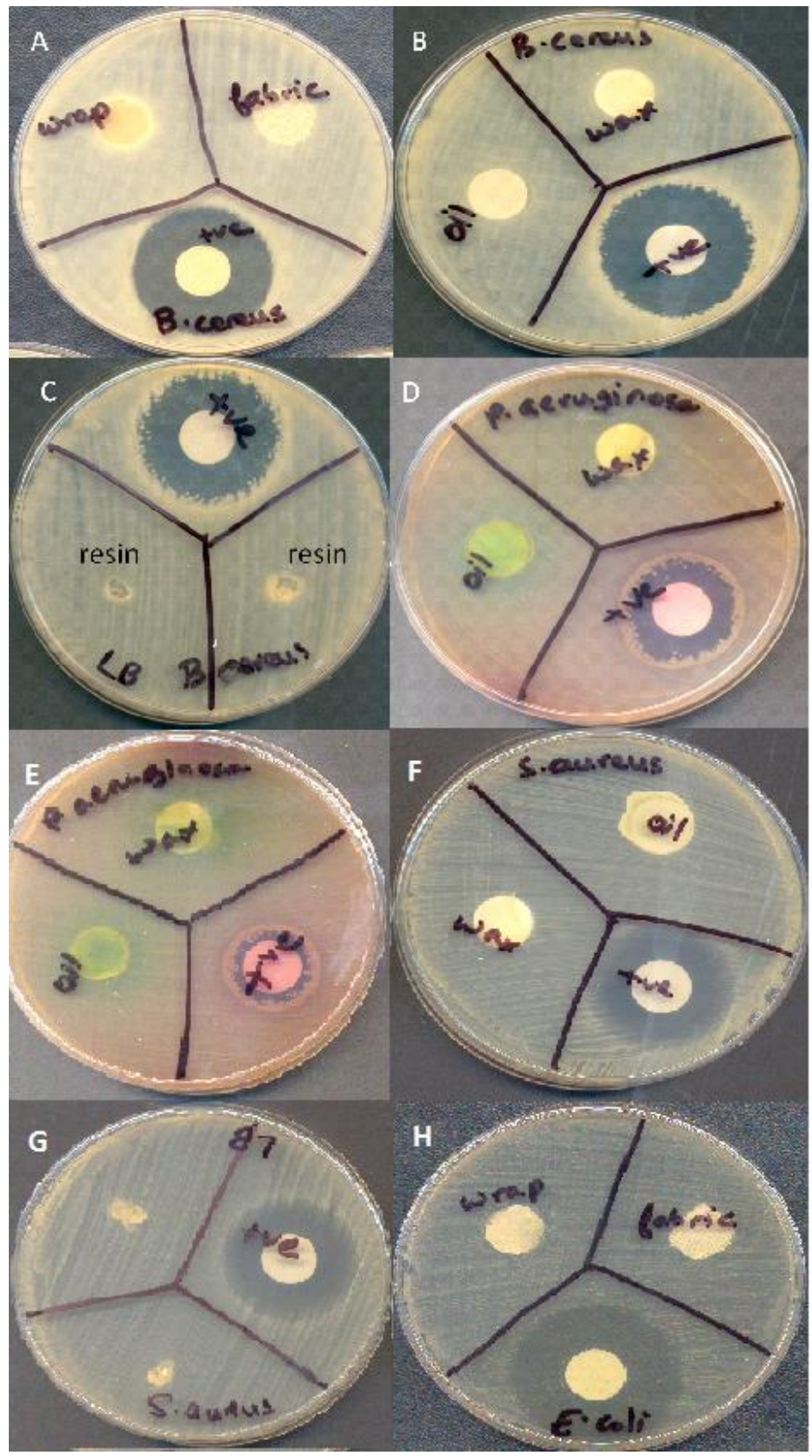

Figure 1 Zone of inhibition assay to test the antibacterial activity of Abeego Fabric and its constituent parts. A) Zone of inhibition assay for Abeego wrap and fabric against $B$. cereus B) Zone of inhibition assay for Abeego oil and wax against $B$. cereus C) Zone of inhibition assay for Abeego resin against $B$. cereus D) Zone of inhibition assay for Abeego oil and wax against $P$. aeruginosa E) Zone of inhibition assay for Abeego oil and wax against $P$. aeruginosa F) Zone of inhibition assay for Abeego oil and wax against $S$. aureus G) Zone of inhibition assay for Abeego resin against S. aureus $\mathrm{H}$ ) Zone of inhibition assay for Abeego wrap and fabric against $E$. coli

\section{Anti-bacterial activity of Abeego wrap}

We decided that the best way to detect the anti-microbial activity of the Abbego wrap was to incubate it with bacterial cells in the liquid phase. To represent the diverse pathogens we have selected both a gram negative bacterium - Salmonella enteritidis and gram positive bacterium -Staphylococcus aureus. S. enteritidis is an organism that is commonly associated with gastrointestinal infections (Feasey et al. 2012). On the other hand $S$. aureus is a commensal inhabiting skin of people and animals. It can however act as an opportunistic pathogen. It can also become a food-borne pathogen when transferred onto dairy products (Fooladi $\boldsymbol{e}$ al. 2010). During the test bacterial cells of both strains were exposed to Abeego 
wrap and its individual components. We discovered that incubation with the wrap has caused a significantly decline in the number of cells of both $S$. enteritidis and $S$. aureus (Fig 2). At the same time some of the individual components of the Abeego wrap also had an impact on the number of observed colony forming units (cfu). However there is no consistency in that manner between both tested organisms. This could mean that each compound has a major effect on a different type of bacterial cell. Therefore the effect of wrap could be attributed to a combined impact of all its components. Overall this confirms the previous suspicion of the anti-bacterial activity of the tested material.

\section{Anti-yeast activity of Abeego wrap}

While the Abeego product shows an effect on bacterial cells it has to be further investigated if it can inhibit the survival of eukaryotic pathogens. For this purpose we have used two strains of Saccharomyces cerevisiae - CRY1 and MYA 3666. S. cerevisiae is the most commonly used representative of Eukarya It serves as a good approximation of fungal food-borne pathogens like Aspergillus flavus (Kim et al. 2004). The yeast cells were treated in a similar manner as $S$. enteritidis and $S$. aureus in the survival assay. The analysis of the number of cfu revealed a small decrease after treatment of CRY1 cells with the Abeego product. However the observed results were not considered to be significant (Fig. 3). This strongly suggests that the tested wrap has no impact on the fitness of eukaryotic cells. It is likely that the composition of yeast membrane and cell wall gives resistance to the effects that were observed in bacteria Therefore similar reaction might be expected from food-borne fungal microorganisms.

\section{Activity on viral capsid}

The last relevant group of food-borne pathogen consists of viruses. The common examples could be noroviruses, rotaviruses as well as hepatitis A and E viruses (Atreya, 2004; Carter, 2005). All of these pathogens possess non-enveloped capsids. On the other hand there are much fewer enveloped viruses that can cause food-borne infections i.e. astroviruses have enveloped viroids and are associated with gastroenteritis (Fleet $\boldsymbol{e t}$ al. 2000). Additionally coronaviruses can cause gastrointestinal symptoms, however these viruses are mostly associated with pneumonic infections (Jevšnik et al., 2013). To investigate the effect of Abeego wrap on viral capsids we tested representatives of non-enveloped viruses. For quick and easy assay we decided to analyse the phage particles. These viruses can be replicated in short time and assayed in a reproducible manner. As viral particles they can act as an approximation of the eukaryotic viruses. The commonly used bacteriophages M13 and P1 were chosen for this experiment The phage particles were tested using similar method as the one used for bacterial and yeast survival assay. Each phage was then enumerated on two-layer plates by infecting E. coli cells. Results show decrease in the overall number of active phage particles (Fig 4). However these changes are not significant enough to support conclusion that the Abeego wrap can inactivate viral particles. A possible explanation for this result would be that the effect of beeswax components is primarily targeting the membrane and therefore has no strong impact on the protein structure of viroid.
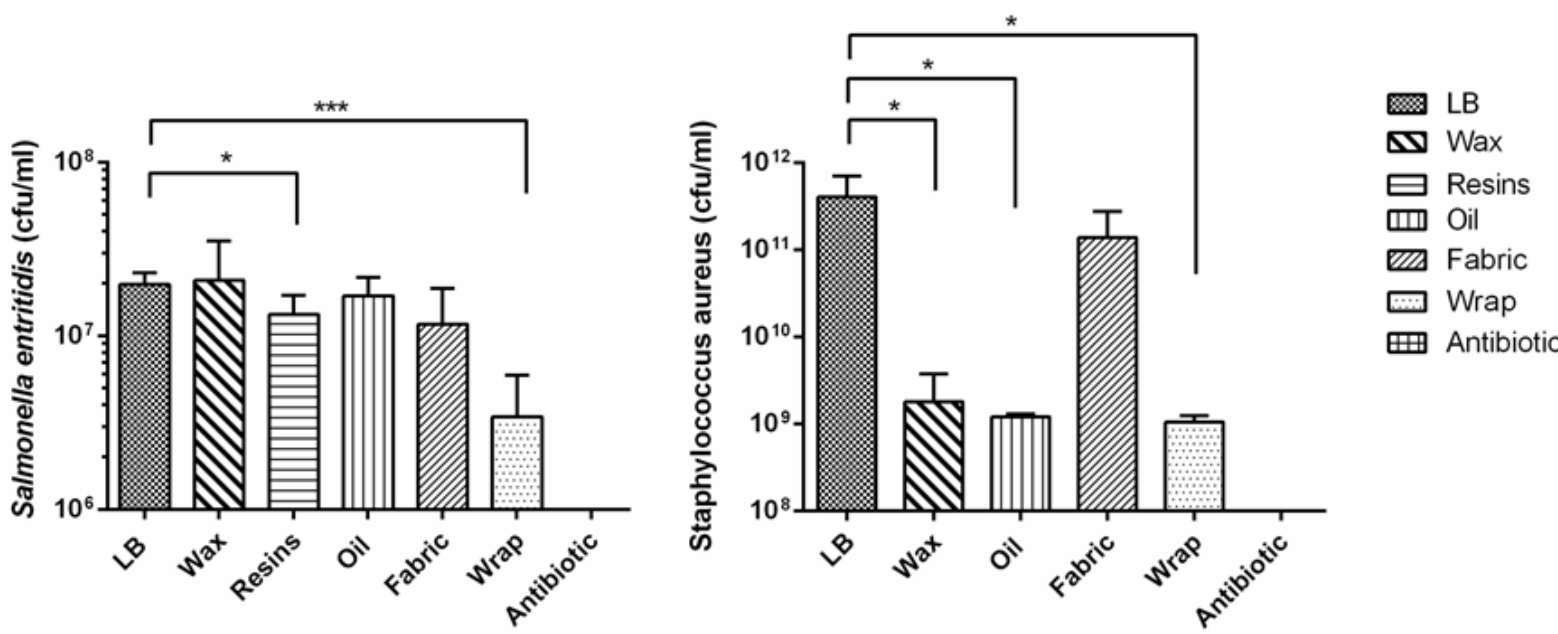

Figure 2 Anti-bacterial activity of Abeego product. The Abeego wrap and its components (wax, resins, oil and fabric) were tested for their anti-microbial effect on cells of Salmonella enteridis and Staphylococcus aureus. Pure LB medium and antibiotic were used as negative and positive control, respectively. Significant decrease in cell number has been observed for $S$. enteridis when comparing LB medium with wrap $(\mathrm{P}=0.002)$ or resins $(\mathrm{P}=0.043)$. Other individual elements of Abeego wrap (wax, oil and fabric) had no significant impact on cell number. Significant decrease in cell number has been observed for $S$. aureus when comparing LB medium with wax $(\mathrm{P}=0.02)$, oil $(\mathrm{P}=0.04)$ or wrap $(\mathrm{P}=0.02)$. The exposure to antibiotic reduced number of cells to zero in both cases. The significance level of $0.05>\mathrm{P}>0.01$ is represented by * symbol on the graph. The significance level of $\mathrm{P}<0.01$ is represented by $* * *$ symbol on the graph. Bars represent standard deviation.
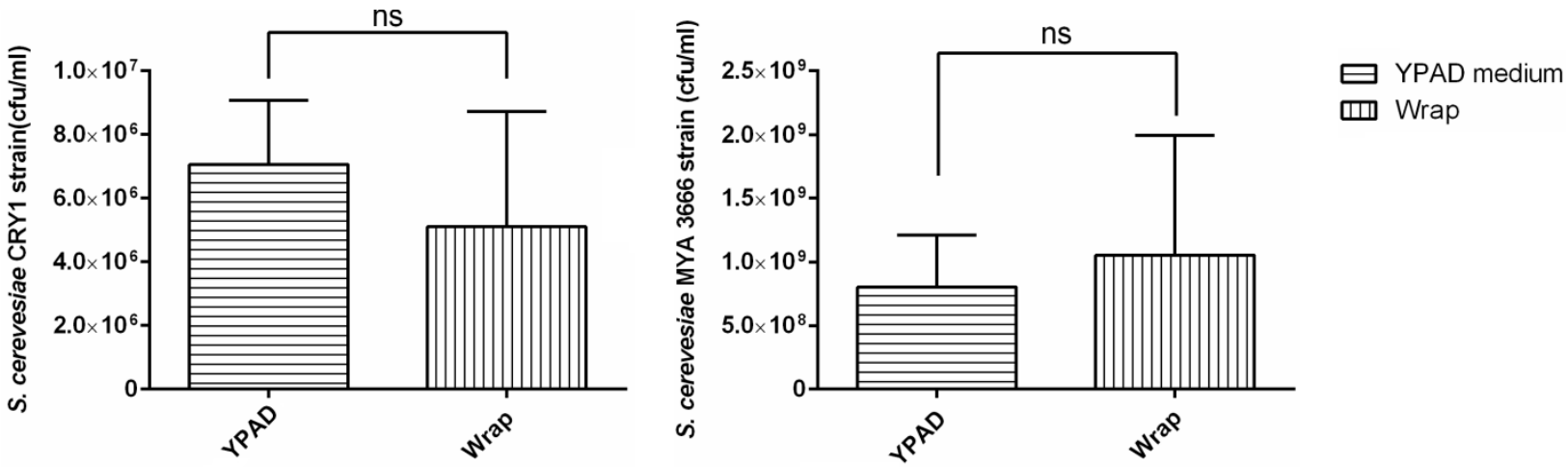

Figure 3 Effect of Abeego product on yeast cells. The Abeego wrap was tested for its anti-microbial effect on cells of Saccharomyces cerevisiae strains CRY1 and MYA 3666. The yeast cells grown in regular YPAD medium were compared to the ones grown in the presence of the wrap. After enumerating cells in both populations no significant differences were observed ( $\mathrm{P}>0.05$, not significant indicated as ns). Bars represent standard deviation. 

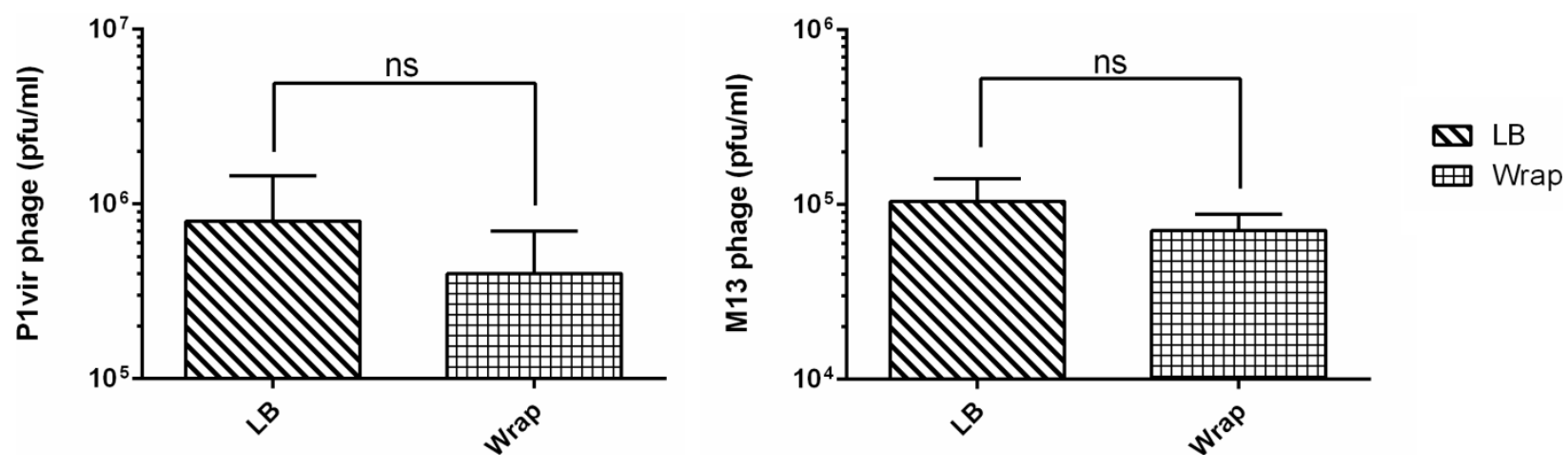

Figure 4 Effect of Abeego product on activity of phage particles. The Abeego wrap was tested for its effect on suspended particles of P1 vir phage and M13 phage. The phages exposed to the wrap and the negative control were enumerated on two-layer plates with appropriate strains of E.coli. No significant differences were observed between the two populations ( $\mathrm{P}>0.05$, not significant indicated as ns). Bars represent standard deviation.

\section{CONCLUSION}

The presented results show that Abeego wrap possesses anti-bacterial activity. This effect can be observed when the microorganisms are exposed to the surface of the wrap, since the active substance cannot diffuse into the environment. The tested product displayed activity against both gram negative and gram positive cells. At the same time this effect does not extend to yeast cells and viral capsids.

Acknowledgments: The work was supported by grant from the Natural Sciences and Engineering Council of Canada, EGP 477804-14. We woud also like to thank Toni Desrosiers for providing resources necessary for this project.

\section{REFERENCES}

Antúnez, K., Harriet, J., Gende, L., Maggi, M., Eguaras, M., \& Zunino, P. (2008). Efficacy of natural propolis extract in the control of American Foulbrood.

Veterinary

Microbiology,

131(3-4),

324-331.

http://dx.doi.org/10.1016/j.vetmic.2008.04.011

Atreya, C. D. (2004). Major foodborne illness causing viruses and current status of vaccines against the diseases. Foodborne Pathogens and Disease, 1(2), 89-96. http://dx.doi.org/10.1089/153531404323143602

Bertani, G. (1951). Studies on lysogenesis. I. The mode of phage liberation by lysogenic Escherichia coli. Journal of Bacteriology, 62(3), 293-300.

Carter, M. J. (2005). Enterically infecting viruses: pathogenicity, transmission and significance for food and waterborne infection. Journal of Applied Microbiology, 98(6), 1354-1380. $\quad$ http://dx.doi.org/10.1111/j.13652672.2005.02635.x

de Andrade Ferreira, F. B., Torres, S. A., da Silva Rosa, O. P., Ferreira, C. M., Garcia, R. B., Marcucci, M. C., \& Gomes, B. P. F. A. (2007). Antimicrobial effect of propolis and other substances against selected endodontic pathogens. Oral Surgery, Oral Medicine, Oral Pathology, Oral Radiology and Endodontology, 104(5), 709-716. http://dx.doi.org/10.1016/j.tripleo.2007.05.019 Feasey, N. A., Dougan, G., Kingsley, R. A., Heyderman, R. S., \& Gordon, M. A. (2012). Invasive non-typhoidal salmonella disease : an emerging and neglected tropical disease in Africa. The Lancet, 379(9835), 2489-2499. http://dx.doi.org/10.1016/s0140-6736(11)61752-2

Fleet, G. H., Heiskanen, P., Reid, I., \& Buckle, K. A. (2000). Foodborne vira illness--status in Australia. International Journal of Food Microbiology, 59(1-2), 127-136. http://dx.doi.org/10.1016/s0168-1605(00)00249-x

Imani Fooladi, A.A., Tavakoli, H.R., \& Naderi, A. (2010). Detection of enterotoxigenic Staphylococcus aureus isolates in domestic dairy products. Iranian Journal of Microbiology, 2(3), 137-42.

Jevšnik, M., Steyer, A., Zrim, T., Pokorn, M., Mrvič, T., Grosek, Š., Strle, F., Lusa, L., \& Petrovec, M. (2013). Detection of human coronaviruses in simultaneously collected stool samples and nasopharyngeal swabs from hospitalized children with acute gastroenteritis. Virology Journal, 10(1), 46. http://dx.doi.org/10.1186/1743-422x-10-46

Kim, J. H., Campbell, B. C., Mahoney, N. E., Chan, K. L., \& Molyneux, R. J. (2004). Identification of phenolics for control of Aspergillus flavus using Saccharomyces cerevisiae in a model target-gene bioassay. Journal of Agricultural and Food Chemistry, 52(26), 7814-7821. http://dx.doi.org/10.1021/jf0487093

Luria, S. E., Adams, J. N., \& Ting, R. C. (1960). Transduction of lactose-utilizing ability among strains of $E$. coli and $S$. dysenteriae and the properties of the transducing phage particles. Virology, 12(3), 348-390. http://dx.doi.org/10.1016/0042-6822(60)90161-6

Malhotra, B., Keshwani, A., \& Kharkwal, H. (2015). Antimicrobial food packaging: potential and pitfalls. Frontiers in Microbiology, 6, 611 http://dx.doi.org/10.3389/fmicb.2015.00611
Marcucci, M.C., Ferreres, F., García-Viguera, C., Bankova, V., S., De Castro, S., L., Dantas, A., P., Valente, P., H., M., \& Paulino, N. (2001). Phenolic compounds from Brazilian propolis with pharmacological activities. Journal of Ethnopharmacology, 74(2), 105-112. http://dx.doi.org/10.1016/s0378 8741(00)00326-3

Messing, J. (1993). M13 Cloning Vehicles: Their Contribution to DNA Sequencing. In DNA Sequencing Protocols (pp. 9-22). New Jersey: Humana Press. http://dx.doi.org/10.1385/0-89603-248-5:9

Murthy, M. S. S., Rao, B. S., Reddy, N. M. S., Subrahmanyam, P., \& Madhvanath, U. (1975). Non-equivalence of YEPD and synthetic complete media in yeast reversion studies. Mutation Research/Fundamental and Molecular Mechanisms of Mutagenesis, 27(2), 219-223. http://dx.doi.org/10.1016/0165 $\underline{1218(75) 90024-5}$

Orsi, R. O., Sforcin, J. M., Rall, V. L. M., Funari, S. R. C., Barbosa, L., \& Fernandes, JR. A. (2005). Susceptibility profile of Salmonella against the antibacterial activity of propolis produced in two regions of Brazil. Journal of Venomous Animals and Toxins Including Tropical Diseases, 11, 109-116. http://dx.doi.org/10.1590/s1678-91992005000200003

Select Committee on GRAS Substances (SCOGS) Opinion: Beeswax (yellow or white). (1975).

Singh, S., ho Lee, M., Park, I., Shin, Y., \& Lee, Y. S. (2016). Antimicrobial seafood packaging: a review. Journal of Food Science and Technology, 53(6), 2505-2518. http://dx.doi.org/10.1007/s13197-016-2216-x

Wilson, M. B., Brinkman, D., Spivak, M., Gardner, G., \& Cohen, J. D. (2015). Regional variation in composition and antimicrobial activity of US propolis against Paenibacillus larvae and Ascosphaera apis. Journal of Invertebrate Pathology, 124, 44-50. http://dx.doi.org/10.1016/j.jip.2014.10.005

Wilson, M. B., Spivak, M., Hegeman, A. D., Rendahl, A., \& Cohen, J. D. (2013) Metabolomics Reveals the Origins of Antimicrobial Plant Resins Collected by Honey Bees. PLoS ONE, 8(10), 1-13. http://dx.doi.org/10.1371/journal.pone.0077512

Zhang, D., \& Xiao, H. (2013). Dual-functional beeswaxes on enhancing antimicrobial activity and water vapor barrier property of paper. ACS Applied Materials and Interfaces, 5, 3464-3468. http://dx.doi.org/10.1021/am400585m 